\title{
Oclusão arterial aguda consequente a embolia paradoxal - relato de caso e revisão de literatura
}

\author{
Acute arterial occlusion resulting from paradoxical embolism - case report and \\ literature review
}

Juliana Daud Ribeiro ${ }^{1}$ (D, Marcio Barreto de Araujo $^{1}$ (D), Barbara de Araujo Casa ${ }^{1}$ (D), João Antonio Correa ${ }^{1}$ (D)

\begin{abstract}
Resumo
A embolia paradoxal é a transposição de um trombo originário da circulação sistêmica venosa para a arterial através de um defeito cardíaco, mais comumente o forame oval pérvio (FOP). A manifestação mais comum é o acidente cerebrovascular. A oclusão arterial aguda (OAA) é rara, requer alta suspeição diagnóstica e corresponde a menos de $2 \%$ de todos casos de embolia arterial. O tromboembolismo pulmonar (TEP) é a causa mais comum de elevação temporária do shunt direita-esquerda em pacientes com FOP e ocorre em pelo menos $60 \%$ dos casos de embolia paradoxal. Em 2019, um homem de 27 anos, sem fator para hipercoagulabilidade, deu entrada no Hospital Universitário do $A B C$, com quadro de OAA grau I Rutherford em membros inferiores secundária a tromboembolismo através de FOP prévio não diagnosticado, associado a trombose venosa profunda de membro inferior direito e TEP bilateral. O manejo incluiu anticoagulação plena e encaminhamento para cirurgia cardíaca.
\end{abstract}

Palavras-chave: embolia; embolia paradoxal; doença arterial periférica; forame oval pérvio; trombose venosa profunda; embolia pulmonar.

\begin{abstract}
Paradoxical embolism describes the passage of a thrombus originating from the systemic venous circulation to the arterial circulation, via an intracardiac defect, usually a patent foramen ovale (PFO). PFOs usually present with stroke. Acute arterial occlusion is rarer, requires a high degree of suspicion for diagnosis, and involves less than $2 \%$ of all cases of arterial embolism. Pulmonary embolism (PE) has been described as the most common cause of temporary elevation of the right-to-left shunt in patients with PFO and occurs in at least 60\% of cases of paradoxical embolism. In 2019, a 27-year-old man with no risk factors for hypercoagulability presented at the Hospital Universitário do ABC, SP, Brazil, with Rutherford grade I acute lower limb arterial occlusion, secondary to thromboembolism through a previously undiagnosed PFO, also associated with deep venous thrombosis of the right lower limb and bilateral PE. Management included full anticoagulation and referral for cardiac surgery.
\end{abstract}

Keywords: embolism; paradoxical embolism; peripheral arterial disease; patent foramen ovale; deep vein thrombosis; pulmonary embolism.

Como citar: Ribeiro JD, Araujo MB, Casa BA, Correa JA. Oclusão arterial aguda consequente a embolia paradoxal relato de caso e revisão de literatura. J Vasc Bras. 2021;20:e20210074. https://doi.org/10.1590/1677-5449.210074

\footnotetext{
${ }^{1}$ Centro Universitário FMABC, Santo André, SP, Brasil. Fonte de financiamento: Nenhuma.

Conflito de interesse: Os autores declararam não haver conflitos de interesse que precisam ser informados. Submetido em: Maio 22, 2021. Aceito em: Agosto 13, 2021.
}

O estudo foi realizado no Hospital de Clínicas, Complexo Hospitalar Municipal de São Bernardo do Campo, pelo serviço de Angiologia e Cirurgia Vascular, Faculdade de Medicina do ABC, Santo André, SP, Brasil. 


\section{INTRODUÇÃO}

O forame oval pérvio (FOP) foi primeiramente descrito por Cohnheim, em 1877, que traçou o caminho de um êmbolo através de um defeito septal no coração. O FOP é uma comunicação entre os átrios comum na vida fetal, porém, ao nascimento e à primeira respiração, o FOP se fecha, pois a pressão do átrio esquerdo se torna maior do que a do átrio direito. A persistência desse forame, potencialmente atravessável por um tromboembolismo, é um defeito estimado em $15-35 \%$ da população adulta, segundo estudos em cadáveres ${ }^{1,2,3}$.

Acidentes cerebrovasculares são a consequência mais comum de embolia paradoxal, sendo rara a oclusão arterial aguda (OAA). Relatamos o caso de um paciente de 27 anos com OAA Rutherford Classe I por um tromboembolismo em artéria femoral comum, artéria femoral, artéria femoral profunda direita e artéria poplítea esquerda devido a um FOP não diagnosticado. O objetivo deste artigo foi auxiliar na praticidade da identificação e manejo dessa doença.

O protocolo foi aprovado pelo Comitê de Ética de nossa instituição (parecer número 4.709.780, CAAE 45679320.8.0000.0082).

\section{RELATO DE CASO}

O paciente de 27 anos, masculino e sem comorbidades, exceto por obesidade grau II (índice de massa corporal $36,2)$, deu entrada em nosso serviço para o tratamento de OAA de membro inferior esquerdo, achado em exame para pesquisa de trombose venosa profunda (TVP) após episódio de tromboembolismo pulmonar bilateral (TEP). O paciente apresentava discreta dor em membros inferiores, ausência de déficit motor ou sensitivo, ausência de gradiente térmico, ausência de pulsos tibiais posteriores bilateralmente, ausência de pulso tibial anterior e poplíteo em membro inferior esquerdo, porém com som audível ao Doppler, e demais pulsos em membro inferior direito diminuídos, o que o classificou como Rutherford Classe I de isquemia aguda.

O interrogatório sobre os diversos aparelhos foi positivo para dispneia aos pequenos esforços, dor e edema importante nos membros inferiores, com piora ao ficar em pé. O paciente negou histórico de tromboembolismo venoso ou pulmonar ou demais moléstias cardíacas. Negou tabagismo ou vícios. A história familiar foi negativa para doença hematológica. Entre 12 irmãos, dois morreram na infância de morte natural não esclarecida, e os demais não apresentaram comorbidades. Os pais eram hipertensos, e a avó materna faleceu por insuficiência cardíaca descompensada.
O paciente referiu viagem recente de ônibus, com duração de 3 dias na ida e na volta e tempo de permanência de 1 mês. Os sintomas iniciaram-se desde o segundo dia de viagem, com claudicação em membro inferior esquerdo aos pequenos esforços, porém não procurou assistência médica durante a estadia. Quatro dias após o retorno, iniciou dispneia associado ao quadro. No oitavo dia, apresentou claudicação no membro inferior direito, não tolerava subir escadas, apresentava frialdade em pododáctilos bilateralmente e melhora da dor em membros inferiores e da dispneia ao repouso. Pela noite, apresentou episódio de síncope, e, na manhã seguinte, foi levado a Unidade de Pronto Atendimento (UPA) por um familiar, tendo sido, após, transferido ao Hospital referência da UPA.

Nesse hospital, o paciente foi admitido taquicárdico e dispneico, com eletrocardiograma evidenciando taquicardia, sinais de sobrecarga de ventrículo direito e inversão de onda $\mathrm{T}$ em parede anterior. Também apresentou ecocardiograma transtorácico evidenciando sinais de disfunção das cavidades direitas, discreta movimentação paradoxal do septo interventricular, sinais indiretos de hipertensão pulmonar e ausência de imagem sugestiva de trombo; além de tomografia positiva para TEP bilateral (Figura 1). Foram realizados anticoagulação plena e ultrassom venoso de membro inferior esquerdo.

Os exames laboratoriais não evidenciaram alterações, incluindo creatinofosfoquinase de $90 \mathrm{U} / \mathrm{L}$. O ultrassom Doppler venoso do membro inferior esquerdo não evidenciou TVP, porém observou-se imagem hipoecoica em topografia de artéria poplítea, na altura da interlinha articular, com ausência de fluxo tanto ao método color quanto à análise espectral, compatível com oclusão da mesma. Após esse achado,

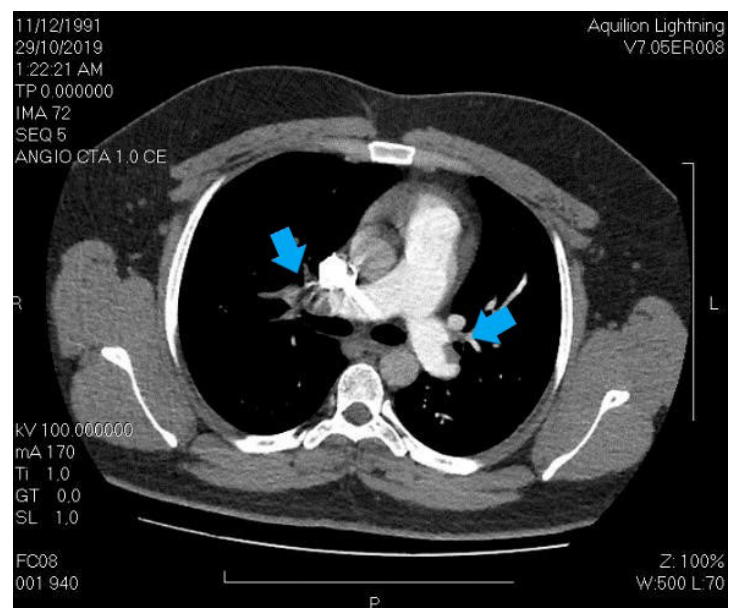

Figura 1. Tomografia protocolo T.E.P., positiva para tromboembolismo pulmonar bilateral (setas azuis). 
o paciente foi encaminhado para a avaliação da equipe de Cirurgia Vascular em nosso serviço.

Em nossa instituição, foi realizado o estudo complementar com os seguintes procedimentos: 1) ultrassom Doppler venoso profundo de membro inferior direito, evidenciando trombose venosa subaguda de veia femoral comum parcialmente (Figura 2) e de veias gastrocnêmias (Figura 3); 2) ultrassom Doppler arterial de membros inferiores pré-angiografia; 3) aortografia sem alterações; 4) arteriografia do membro inferior direito, que mostrou artéria femoral profunda com imagem sugestiva de trombo em um de seus ramos (Figura 4), artéria poplítea e infrapatelares com irregularidades parietais, artéria tibial anterior ocluída na transição com a artéria pediosa e artéria fibular ocluída em terço médio sem reenchimento, com arco plantar incompleto; 5) arteriografia de membro inferior esquerdo, com artéria poplítea ocluída em segmento suprapatelar sem reenchimento, evidenciando tronco tibiofibular com reenchimento logo antes da sua

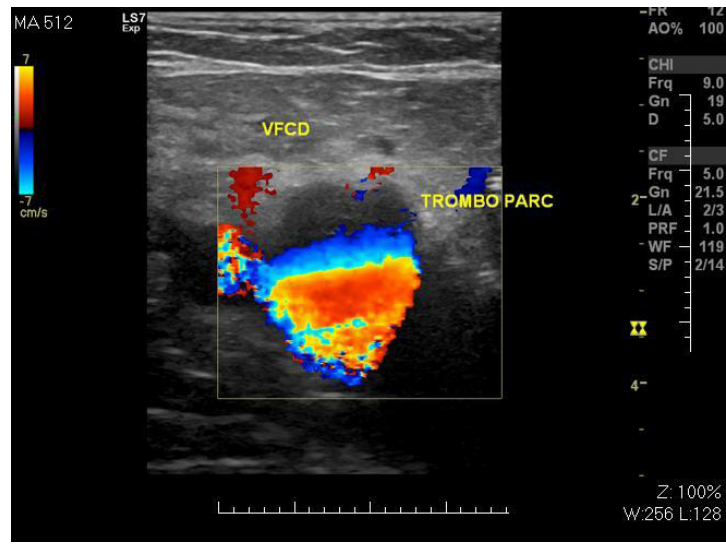

Figura 2. Trombose venosa subaguda de veia femoral comum parcialmente.

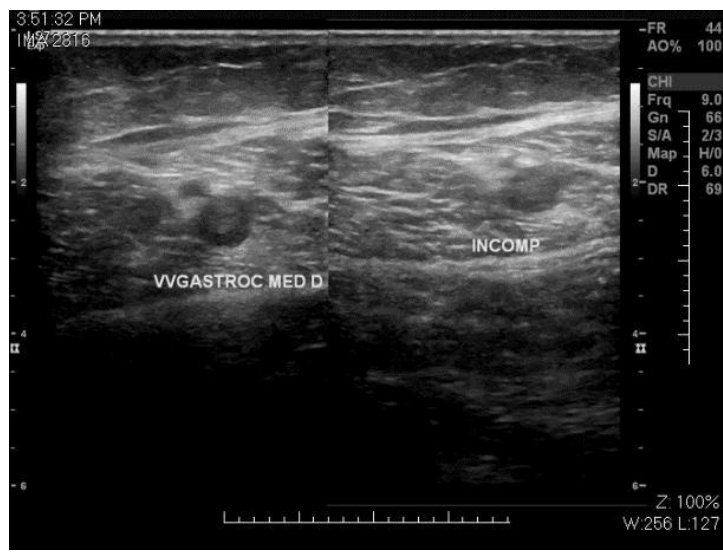

Figura 3. Trombose venosa subaguda de veias gastrocnêmias. bifurcação (Figura 5), artéria tibial anterior e artéria tibial posterior ocluídas na origem sem reenchimento, artéria fibular pérvia, com estenoses menores que $50 \%$ e irregularidades parietais e arco plantar não contrastado; 6) ecocardiograma transesofágico, com FOP (bordas do forame $>5 \mathrm{~mm}$ ), septo interatrial com descolamento da fossa oval de $0,25 \mathrm{~mm}$ e túnel longo de $13 \mathrm{~mm}$ e ausência de trombos ou vegetações (Figura 6); e 7) provas de trombofilia negativas.

Foi instituído o tratamento conservador com anticoagulação plena com enoxaparina durante a internação. Após o período de internação, o paciente evoluiu com pulsos presentes e cheios em membro inferior direito com índice tornozelo-braquial

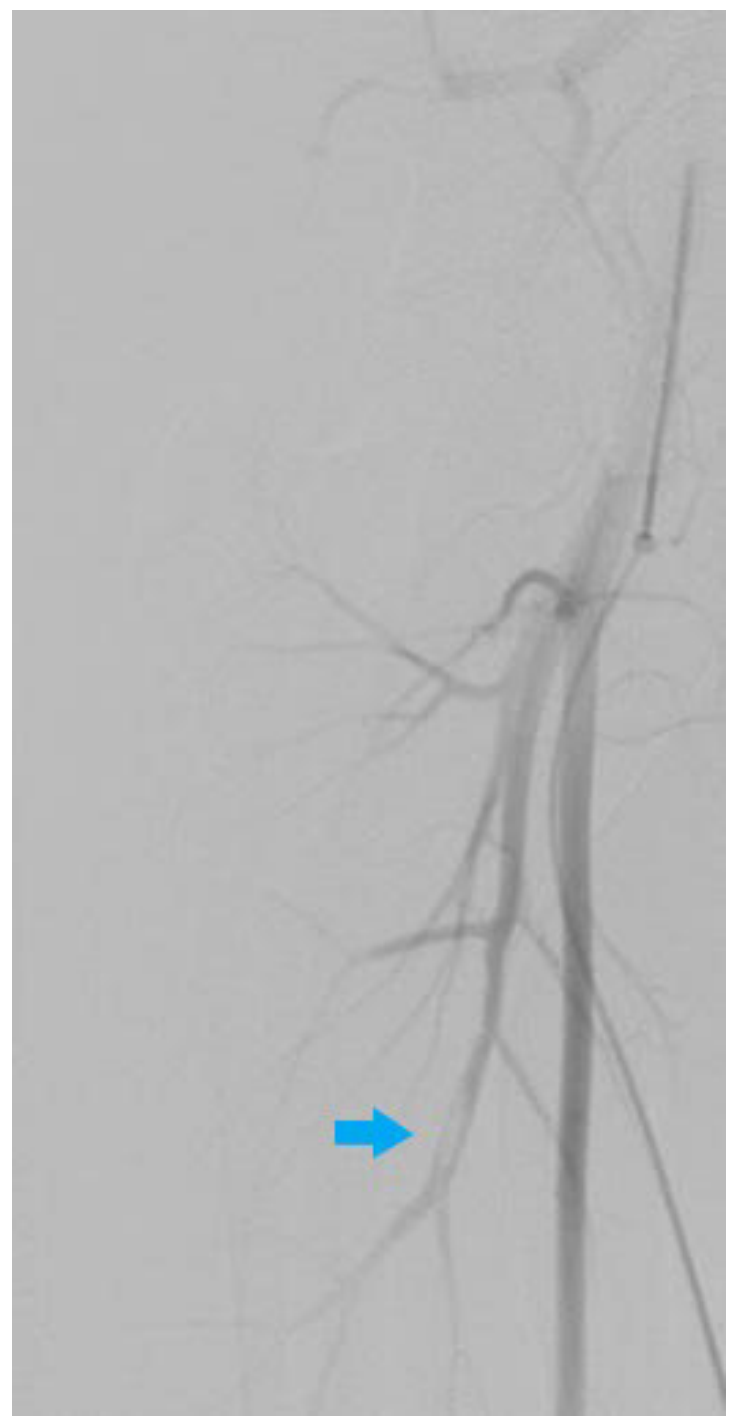

Figura 4. Fotografia de angiografia com subtração digital que demonstra artéria femoral profunda com imagem sugestiva de trombo em um de seus ramos (seta azul). 


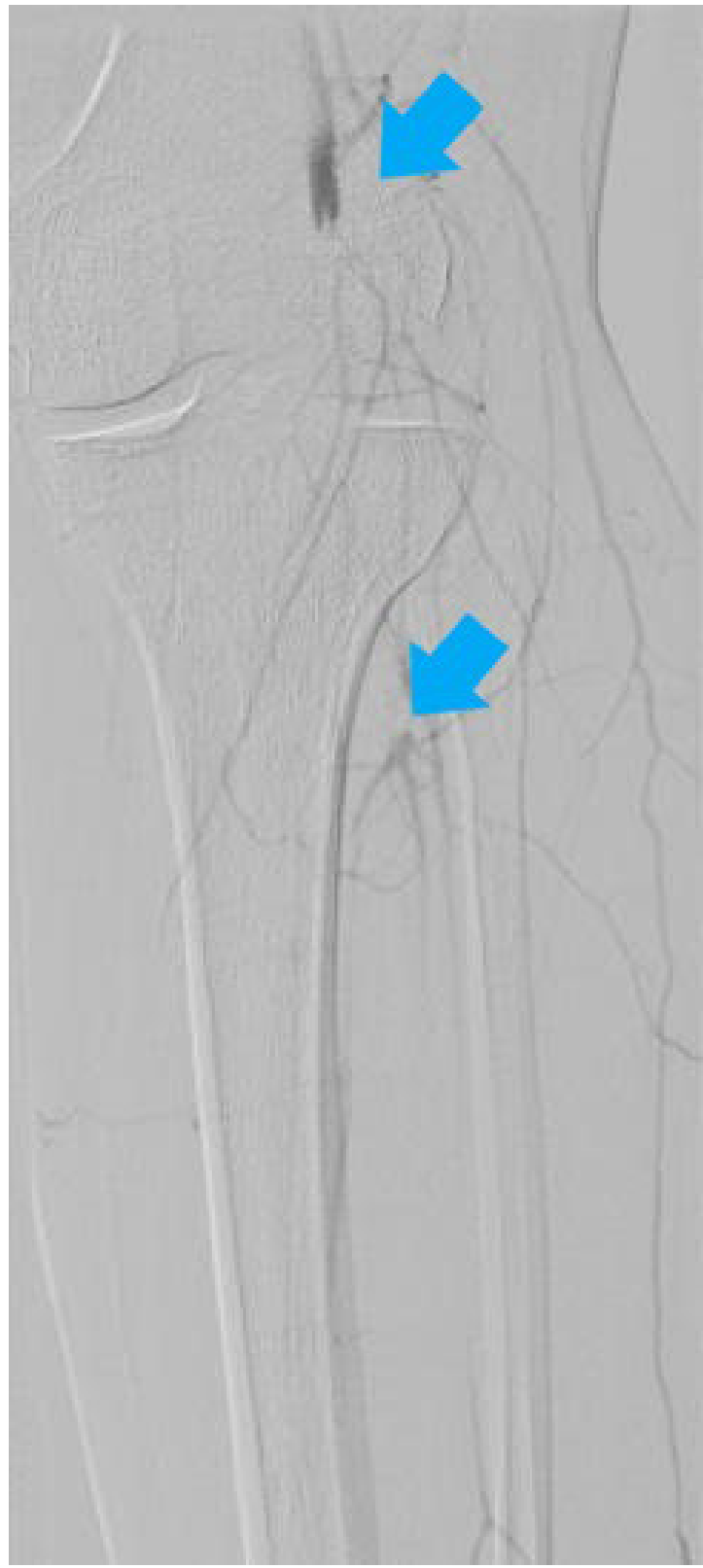

Figura 5. Fotografia de angiografia com subtração digital que demonstra artéria poplítea ocluída em segmento suprapatelar sem reenchimento (seta azul superior) e tronco tibiofibular com reenchimento logo antes da sua bifurcação (seta azul inferior).

(ITB) $>1,0$ e com surgimento de pulso poplíteo diminuído em membro inferior esquerdo, apesar de manter a ausência de pulsos distais, com ITB de 0,53 . O paciente teve alta hospitalar com edoxabana $60 \mathrm{mg} \mathrm{1x/dia} \mathrm{e} \mathrm{encaminhamento} \mathrm{para} \mathrm{programação}$ ambulatorial de correção do FOP com a cirurgia cardíaca. Não foram feitos exames de imagem no seguimento devido à pandemia de covid-19.

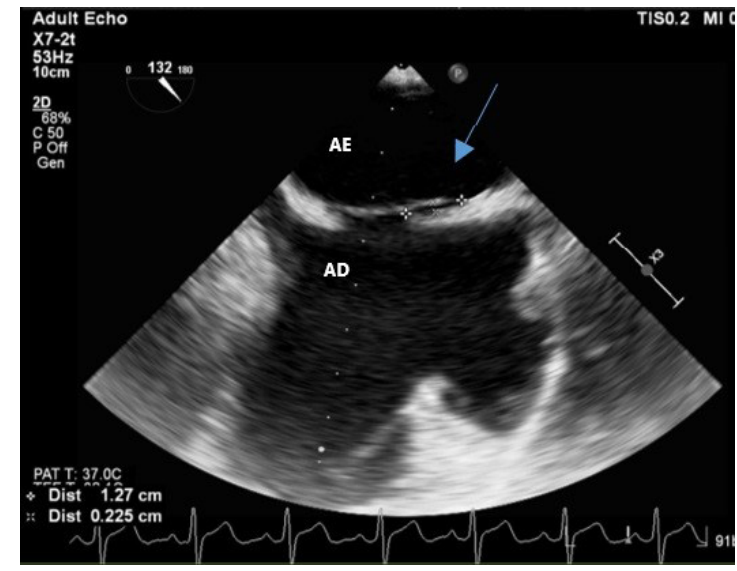

Figura 6. Forame oval pérvio (seta azul). AD: átrio direito; AE: átrio esquerdo.

\section{DISCUSSÃO}

Não foi encontrado outro relato semelhante na literatura nacional. Em um estudo no programa de cardiologia intervencionista da Universidade da Califórnia, 416 pacientes foram referenciados, entre 2001 e 2009, para o fechamento do FOP. Destes, apenas $4(0,96 \%)$ apresentaram embolia arterial periférica, sendo um para a artéria poplítea, dois para a artéria oftálmica e um para a artéria braquial ${ }^{4}$.

Entre 1989 e 1999, em um estudo retrospectivo, a embolia paradoxal foi responsável por 13 casos de OAA. Em um serviço grande de Cirurgia Vascular seria esperado que pelo menos um paciente por ano fosse diagnosticado com essa etiologia para $\mathrm{OAA}^{5}$.

Para que ocorra a embolia paradoxal, o shunt deve ocorrer no momento específico em que o trombo se encontre no átrio direito, visto que não basta o paciente ter o FOP - deve ocorrer o shunt direita-esquerda pelo forame. O shunt direita-esquerda pode ocorrer com o aumento do volume ou da pressão intratorácica ${ }^{6}$.

Um estudo sobre a prevalência de TVP em pacientes com suspeita de embolia paradoxal concluiu que, quando o FOP foi detectado nos pacientes com embolismo, foi frequente a presença de trombose venosa oculta em membros inferiores. Nesse mesmo estudo, 264 pacientes com suspeita de eventos embólicos foram submetidos ao ecocardiograma transesofágico. Destes, 49 pacientes (24 mulheres e 25 homens) tinham FOP, dos quais 41 apresentaram acidente vascular cerebral e 8 tiveram $\mathrm{OAA}^{7}$.

Em outro estudo, os autores questionaram se a embolia paradoxal e a OAA eram eventos raros ou pouco suspeitados e sugeriram que pacientes com OAA devessem ser submetidos a angiografia e, caso não houvesse fonte embólica, fossem submetidos ao 
ecocardiograma transesofágico. Caso fosse diagnosticado o FOP com shunt direita-esquerda e houvesse evidência de TVP ou embolia paradoxal, deveria ser realizada a correção do FOP. Caso a evidência fosse negativa, o FOP deveria ser fechado nos casos de eventos significativos ou recorrentes, e o filtro de veia cava e/ou a varfarina seriam as terapias preconizadas ao longo de 12 meses, seguidas de regime antiplaquetário (Grau de Recomendação C) ${ }^{8,9,10}$.

A idade média de pacientes com embolia paradoxal secundária a FOP é de 54 anos, acometendo igualmente homens e mulheres. Em sua maioria, acomete os membros inferiores, principalmente o membro inferior esquerdo. Os casos com múltiplos membros afetados são infrequentes, e TVP e TEP são comumente associados. O manejo inicial deve ser igual ao de outras OAA. A maioria dos pacientes necessita ser submetida a tromboembolectomia de urgência e/ou fibrinólise, com ou sem fasciotomia, e a amputação raramente ocorre ${ }^{1,11}$.

Os exames complementares incluem eletrocardiograma, ecocardiograma transtorácico ou transesofágico, ultrassom Doppler venoso profundo de membros inferiores caso haja clínica sugestiva ou após a identificação de FOP e investigação de trombofilias (Grau de Recomendação C) $)^{1,12,13}$.

No caso apresentado, não foi necessária abordagem cirúrgica e houve acometimento de múltiplos membros, correspondendo a uma minoria em relação à literatura, como também o paciente em questão era jovem em relação à faixa etária mais acometida. $\mathrm{O}$ fato de uma viagem com duração de 3 dias ter sido realizada duas vezes em um período de 1 mês, dentro de um ônibus com poucas paradas e pouca mobilidade, foi um fator importante para predispor a formação de trombo na circulação venosa, e, por não haver outras etiologias mais prováveis, foi fator crucial para a suspeita de embolia paradoxal.

O tempo de anticoagulação proposto pela nossa equipe foi de 6 meses ou até a correção do FOP. Não há fatores preditivos para identificar ou quantificar o risco de embolia paradoxal em shunts cardíacos previamente diagnosticados.

A embolia é, em mais de $80 \%$ dos casos, consequência do tromboembolismo cardíaco. Porém, em pacientes em que a OAA não tem etiologia definida, o diagnóstico diferencial com embolia paradoxal secundário a FOP deve ser considerado ${ }^{1,13,14,15}$. Este artigo tem o intuito de contribuir e expandir o conhecimento sobre o assunto, bem como aumentar a suspeição dessa rara doença.

\section{REFERÊNCIAS}

1. GreenbergJW, Goff ZD, Mooser AC, Wittgen CM, Smeds MR. Acute limb ischemia secondary to patent ovale-mediated paradoxal embolism: case report and systematic review of the literature. Ann Vasc Surg. 2020;66:668.e5-10. http://dx.doi.org/10.1016/j. avsg.2019.12.022. PMid:31904517.

2. Lagattolla NR, Sandison AJ, Smith K, Cochrane GM, Chambers J, Taylor PR. Acute limb ischemia due to paradoxical embolism from a long-term central venous catheter. Eur J Vasc Endovasc Surg. 1998;16(4):365-6. http://dx.doi.org/10.1016/S1078-5884(98)800592. PMid:9818017.

3. Miller S, Causey MV, Schachter D, Andersen CA, Singh N. A case of limb ischemia secundary to paradoxical embolism. Vasc Endovascular Surg. 2010;44(7):604-8. http://dx.doi.org/10.1177/1538574410374656. PMid:20675331.

4. Dao CN, Tobis JM. PFO and paradoxical embolism producing events other than stroke. Catheter Cardiovasc Interv. 2011;77(6):903-9. http://dx.doi.org/10.1002/ccd.22884. PMid:21207422.

5. Travis JA, Fuller SB, Ligush J Jr, Plonk GW Jr, Geary RL, Hansen KJ. Diagnosis and treatment of paradoxical embolus. J Vasc Surg. 2001;34(5):860-5. http://dx.doi.org/10.1067/mva.2001.118815. PMid:11700487.

6. Pinard EA, Fazal S, Schussler JM. Catastrophic paradoxical embolus after hemodialysis acces thrombectomy in a patient with a patent foramen ovale. Int Urol Nephrol. 2013;45(4):1215-7. http://dx.doi. org/10.1007/s11255-012-0141-0. PMid:22367171.

7. Stöllberger C, Slany J, Schuster I, Leitner H, Winkler WB, Karnik $\mathrm{R}$. The prevalence of deep venous thrombosis in patientes with suspected paradoxical embolism. Ann Intern Med. 1993;119(6):4615. http://dx.doi.org/10.7326/0003-4819-119-6-199309150-00003. PMid:8357111.

8. Chaik of E, Campbell BE, Smith RB 3rd. Paradoxal embolism and acute arterial occlusion: rare or unsuspected? J Vasc Surg. 1994;20(3):377-84. http://dx.doi.org/10.1016/0741-5214(94)90135-X. PMid:8084029.

9. Windecker S, Stortecky S, Meier B. Paradoxical embolism. J Am Coll Cardiol. 2014;64(4):403-15. http://dx.doi.org/10.1016/j. jacc.2014.04.063. PMid:25060377.

10. Capilla-Montes MT, Gómez-Caravaca J, Fernández-Catalán C, Landaluce Chaves M, Mira-Sirvent F, Masegosa-Medina JA. Embolismo Paradójico: uma entidade infrecuente o poco diagnosticada? Angiologia. 2007;59(6):451-6. http://dx.doi. org/10.1016/S0003-3170(07)75082-4.

11. Hagen PT, Scholz DG, Edwards WD. Incidence and size of patent foramen ovale during the first 10 decades of life: an autopsy study of 965 normal hearts. Mayo Clin Proc. 1984;59(1):17-20. http:// dx.doi.org/10.1016/S0025-6196(12)60336-X. PMid:6694427.

12. Abad-Arranz M, Jara-Palomares L, Martos-Maine JL, CarrascoHernandez L, Ortega-Ruiz F, Otero-Candelera R. Tromboembolismo Pulmonar y embolia paradójica concomitante: a propósito de um caso. Arch Bronconeumol. 2014;50(3):120-2. http://dx.doi. org/10.1016/j.arbres.2013.05.008. PMid:24491889.

13. Hakman EN, Cowling KM. Paradoxical embolism [site na Internet]. Treasure Island (FL): StatPearls Publishing; 2021. https://www.ncbi. nlm.nih.gov/books/NBK470196/

14. Castilla-Carretero JJ, González-Arranz MA, Fustero-Aznar JM, et al. Isquemia aguda por embolia paradójica. Angiologia. 2004;56(6):5718. http://dx.doi.org/10.1016/S0003-3170(04)74910-X.

15. Ahmed S, Siddiqui AK, Mattana J, Sadiq A, Borgen E. Paradoxal arterial emboli causing acute limb ischemia in a patient with essential thrombocytosis. Am J Med Sci. 2003;326(3):156-8. http:// dx.doi.org/10.1097/00000441-200309000-00011. PMid:14501234. 
Correspondência Juliana Daud Ribeiro

Av. Onze de Junho, 1070, Cj 1207 - Vila Clementino CEP 04041-004 - São Paulo (SP), Brasil Tel.: (11) 97423-5907 E-mail: julianadaudr@gmail.com

Informações sobre os autores JDR - Médica Cirurgiã Vascular, Centro Universitário FMABC. MBA - Assistente em Cirurgia Vascular, Centro Universitário FMABC. BAC - Acadêmica de Medicina, Centro Universitário FMABC. JAC - Professor Titular; Assistente em Cirurgia Vascular, Centro Universitário FMABC

Contribuições dos autores Concepção e desenho do estudo: JDR, JAC Análise e interpretação dos dados: JDR, MBA, JAC Coleta de dados: JDR, BAC Redação do artigo: JDR, BAC Revisão crítica do texto: JDR, MBA, JAC Aprovação final do artigo: JDR, MBA, BAC, JAC Análise estatística: JDR, MBA, JAC Responsabilidade geral pelo estudo: JDR, MBA, BAC, JAC

*Todos os autores leram e aprovaram a versão final submetida ao J Vasc Bras. 